\title{
Durable Superhydrophobic and Antireflective Surfaces by Trimethylsilanized Silica Nanoparticles-Based Sol-Gel Processing
}

\author{
Michele Manca, ${ }^{* \dagger}$ Alessandro Cannavale, ${ }^{\dagger}$ Luisa De Marco ${ }^{\dagger}$ Antonino S. Aricò, ${ }^{\S}$ \\ Roberto Cingolani, ${ }^{\dagger}$ and Giuseppe Gigli ${ }^{\dagger}$ \\ ${ }^{\dagger}$ National Nanotechnology Laboratories of CNR-INFM c/o Istituto Superiore Universitario di Formazione \\ Interdisciplinare, sez. Nanoscienze, Distretto Tecnologico, Università del Salento, Via Arnesano, 16, 73100 \\ Lecce, Italy, ${ }^{\circledR}$ Politecnico di Bari, Dipartimento di Architettura e Urbanistica, Via E. Orabona, 4, 70126 Bari, \\ Italy, and ${ }^{\S}$ CNR-ITAE Institute, via Salita S. Lucia sopra Contesse, 5, 98126 Messina, Italy
}

Received December 9, 2008. Revised Manuscript Received February 8, 2009

\begin{abstract}
We present a robust and cost-effective coating method to fabricate long-term durable superhydrophobic and-simultaneously-antireflective surfaces by a double-layer coating comprising trimethylsiloxane (TMS) surfacefunctionalized silica nanoparticles partially embedded into an organosilica binder matrix produced through a sol-gel process. A dense and homogeneous organosilica gel layer was first coated onto a glass substrate, and then, a trimethylsilanized nanospheres-based superhydrophobic layer was deposited onto it. After thermal curing, the two layers turned into a monolithic film, and the hydrophobic nanoparticles were permanently fixed to the glass substrate. Such treated surfaces showed a tremendous water repellency (contact angle $=168^{\circ}$ ) and stable self-cleaning effect during $2000 \mathrm{~h}$ of outdoor exposure. Besides this, nanotextured topology generated by the self-assembled nanoparticles-based top layer produced a fair antireflection effect consisting of more than a $3 \%$ increase in optical transmittance.
\end{abstract}

\section{Introduction}

Learning from nature is an important source of new techniques and advanced materials. A typical example of this is the creation of superhydrophobic surfaces by bionic copying of the microstructure of lotus leaves. ${ }^{1,2}$ The superhydrophobic behavior of lotus leaves, known as the lotus leaf effect or self-cleaning effect, is found to be a result of the hierarchical rough structure, as well as the wax layer present on the leaf surface. It has been wellestablished that the wettability of a solid surface is governed by both the surface chemical composition and the surface geometrical microstructures. ${ }^{3-5}$ To fabricate a surface with a water contact angle (CA) larger than $150^{\circ}$, these two key factors must be considered. When the surface energy is lowered, the hydrophobicity is enhanced. However, even the material with the lowest surface energy gives a water CA of only around $120^{\circ} .{ }^{6}$ Generally, very large CAs and a superhydrophobic behavior can be determined only if low surface energy and elevated roughness are simultaneously present.

In the past decade, there have been many attempts at preparing superhydrophobic surfaces by mimicking, in different ways, the lotus leaf surface structure. Most of the preparations involve strict

*To whom correspondence should be addressed. E-mail: michele.manca@ unile.it.

(1) Sun, T. L.; Feng, L.; Gao, X. F.; Jiang, L. Acc. Chem. Res. 2005, 38, 644.

(2) Sun, M. H.; Luo, C. X.; Xu, L. P.; Ji, H.; Qi, Q. Y.; Yu, D. P.; Chen, Y. Langmuir 2005, 21, 8978.

(3) Marmur, A. Langmuir 2004, 20, 3517

(4) Barthlott, W.; Neinhuis, C. Planta 1997, 202, 1.

(5) Manca, M.; Cortese, B.; Viola, I.; Aricò, A. S.; Cingolani, R.; Gigli, G. Langmuir 2008, 24, 1833.

(6) Nishino, T.; Meguro, M.; Nakamae, K.; Matsushita, M.; Ueda, Y. Langmuir 1999, 15,4321

(7) Hozumi, A.; Takai, O. Thin Solid Films 1997, 303, 222.

(8) Han, J. T.; Xu, X. R.; Cho, K. W. Langmuir 2005, 21, 6662.

(9) Shirtcliffe, N. J.; McHale, G.; Newton, M. I.; Perry, C. C. Langmuir 2003 19,5626 . conditions such as harsh chemical treatments, ${ }^{7-9}$ expensive materials (e.g., fluoroalkylsilanes ${ }^{10-12}$ and nanotubes ${ }^{13}$ ), and complex processing procedures including plasma etching, ${ }^{14,15}$ chemical vapor deposition, ${ }^{16,17}$ electrodeposition, ${ }^{18}$ calcinations, ${ }^{19}$ and the use of templates. ${ }^{20-22}$ Many of the reported superhydrophobic polymeric films are often composed of un-cross-linked, thermoplastic polymers, and they are vulnerable to environmental attacks (e.g., solvents and heat). Moreover, it is very difficult to control and reproduce dual size surface roughness in a hierarchical manner with structures smaller than $100 \mathrm{~nm}$ by conventional technologies. Finally, as result of the patterning, such hydrophobic surfaces usually strongly scatter light and produce losses in the optical transparency. As a consequence of these limiting features, in most cases, practical application of superhydrophobic films has been strongly limited.

Little research has been so far focused on the development of a simple way of fabricating transparent superhydrophobic surfaces

(10) Shibuichi, S.; Yamamoto, T.; Onda, T.; Tsujii, K. J. Colloid Interface Sci. 1998, 208, 287.

(11) Hou, H.; Chen, Y. J. Sol-Gel Sci. Technol. 2007, 43, 53.

(12) Yamanaka, M.; Sada, K.; Miyata, M.; Hanabusa, K.; Nakano, K. Chem. Commun. 2006, 2248.

(13) Lau, K. S.; Bico, J.; Teo, K. B.; Chhowalla, M.; Amaratunga, G. A.; Milne, W. I.; McKinley, G. H.; Gleason, K. K. Nano Lett. 2003, 3, 1701.

(14) Ogawa, K.; Soga, M.; Takada, Y.; Nakayama, I. Jpn. J. Appl. Phvs. 1993, 32, L614.

(15) Olde Riekerink, B. M.; Terlingen, J. G. A.; Engbers, G. H. M.; Feijen, J. Langmuir 1999, 15, 4847.

(16) Huang, L.; Lau, S. P.; Yang, H. Y.; Leong, E. S.; Yu, S. F.; Prawer, S. J. Phys. Chem. B 2005, 109, 7746.

(17) Tadanaga, K.; Kitamuro, K.; Matsuda, A.; Minami, T. J. Sol-Gel Sci. Technol. 2003, 26, 705 .

(18) Shi, F.; Wang, Z.; Zhang, X. Adv. Mater. 2005, 17, 1005.

(19) Nakajima, A.; Fujishima, A.; Hashimoto, K.; Watanabe, T. Adv. Mater. 1999, 16, 1365.

(20) Bico, J.; Marzolin, C.; Quere, D. Europhys. Lett. 1999, 47, 220.

(21) He, B.; Patankar, N. A.; Lee, J. Langmuir 2003, 19, 4999.

(22) Sun, S.; Luo, C.; Xu, L.; Ji, H.; Quyang, Q.; Yu, Y.; Chen, Y. Langmuir 2005, 21, 8978 . 


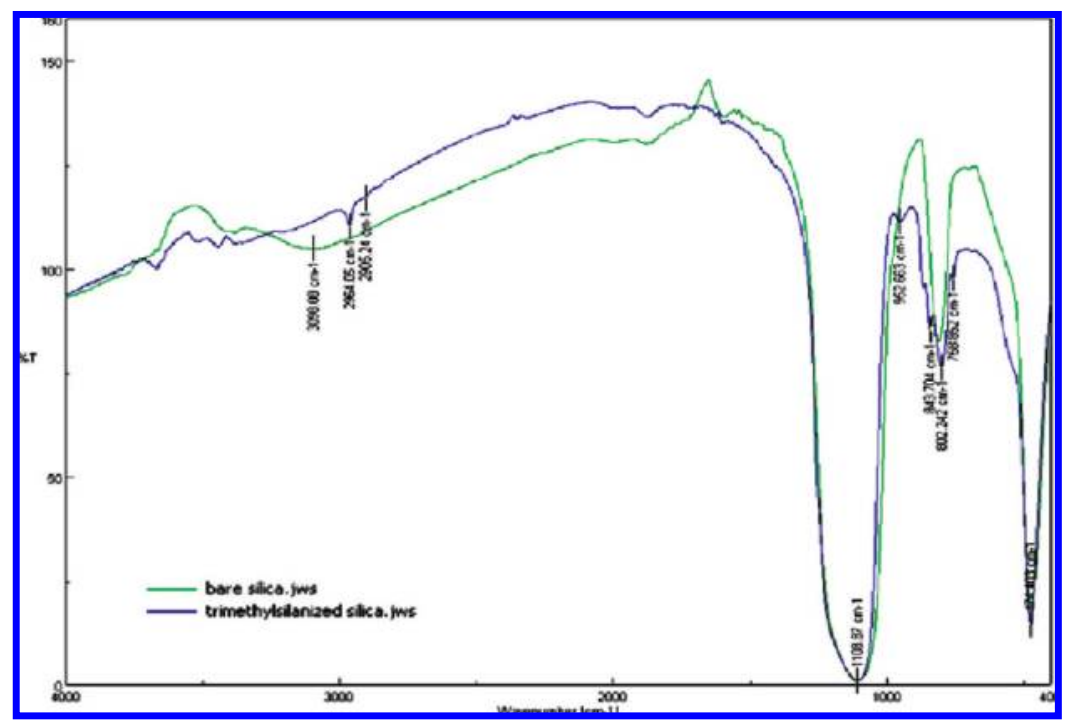

Figure 1. FT-IR spectra of bare and trimethylsilanized silica nanospheres.

for lighting and photonics applications. Some authors ${ }^{23-26}$ reported transparent superhydrophobic coatings by using a dispersion of silica (or titanium dioxide) nanoparticles to create an appropriate surface roughness and a fluorinated cover layer to modify the surface chemical nature. However, in spite of using such low surface energy cover layers, the CA of these films was not very high $\left(<150^{\circ}\right)$, and the sliding angle (SA) was higher than $10^{\circ}$. Bravo et al. ${ }^{27}$ recently demonstrated highly transparent superhydrophobic films by a layer-by-layer process with $\mathrm{SiO}_{2}$ nanoparticles of various sizes.

In all of these cases, the idea has been to create a high aspect ratio topographic surface and to then apply a thin (usually a monolayer) hydrophobic coating. Typically, the hydrophobic coating provides a CA of $115-120^{\circ}$ on a flat surface, and the topography then enhances this to $150-180^{\circ}$. However, a similar approach cannot be successfully extended to large-scale production due to its inadequacy to guarantee chemical and thermal long-term stability as well as to the high cost of fluorinated materials. Therefore, much of the current work is materials oriented with attempts to create hard, transparent, and self-cleaning surfaces.

Starting from these remarks, we have been focused on the development of long-term durable transparent superhydrophobic surfaces via simple coating methods that can simultaneously construct a solid surface having both appropriate roughness and low surface energy without any additional hydrophobic capping layer. In this work, we present an easy and efficient approach to generate robust superhydrophobic surfaces exhibiting a self-cleaning behavior by simply depositing a sol-gel-based coating containing trimethylsiloxane (TMS) surface-functionalized silica nanoparticles onto a precoated silica gel layer.

\section{Experimental Section}

2.1. Preparation of TMS-Silica Nanoparticles. To obtain the TMS surface-functionalized silica nanoparticles, $3 \mathrm{~g}$ of Aerosil 150 (from Evonik-Degussa) was dispersed within $50 \mathrm{~mL}$ of

(23) Soeno, T.; Inokuchi, K.; Shiratori, S. Appl. Surf. Sci. 2004, 237, 543.

(24) Hikita, M.; Tanaka, K.; Nakamura, T.; Kajiyama, T.; Takahara, A. Langmuir 2005, 21, 7299.

(25) Nakajima, A.; Abe, K.; Hashimoto, K.; Watanabe, T. Thin Solid Films $\mathbf{2 0 0 0}, 376,140$

(26) Duparre, A.; Flemming, M.; Steinert, J.; Reihs, K. Appl. Opt. 2002 $41,3294$.

(27) Bravo, J.; Zhai, L.; Wu, Z.; Cohen, R. E.; Rubner, M. F. Langmuir 2007 27,7293 . methyl-isobutyl-ketone (MIK; 99.0\%, from Sigma-Aldrich) and added to a vessel containing $100 \mathrm{~mL}$ of reagent grade hexamethyldisilazane (HMDS) (99.0\%, from Sigma-Aldrich). The solution was placed at $50{ }^{\circ} \mathrm{C}$ and stirred for $24 \mathrm{~h}$ under an $\mathrm{N}_{2}$ inert atmosphere. The particles were then washed three times by diethyl ether and dried at $150{ }^{\circ} \mathrm{C}$ for $5 \mathrm{~min}$. This procedure was also reported by other authors. ${ }^{28}$ The existence of TMS groups grafted onto the silica nanoparticles surface was confirmed by Fourier transform infrared (FT-IR) analysis of both bare and HMDStreated silica that are reported in Figure 1.

Both spectra showed a very strong absorption band at $1108 \mathrm{~cm}^{-1}$ assigned to the stretching modes of the $\mathrm{Si}-\mathrm{O}-\mathrm{Si}$ groups, whereas the bending vibrations of these groups correspond to the strong band observed at $474 \mathrm{~cm}^{-1}$. The weak, sharp absorption mode at $3663-3667 \mathrm{~cm}^{-1}$ is associated with hydroxyl stretching of $\mathrm{SiOH}$ and the medium intensity band at $801 \mathrm{~cm}^{-1}$ to $\mathrm{Si}-\mathrm{O}$ stretching vibration. ${ }^{29}$ In the spectrum of modified silica, further absorption peaks were revealed at 2963 and $2905 \mathrm{~cm}^{-1}$, ascribed, respectively, to antisymmetric and symmetric $\mathrm{C}-\mathrm{H}$ stretching modes, and $758 \mathrm{~cm}^{-1}$, corresponding to $\mathrm{Si}\left(\mathrm{CH}_{3}\right)_{3}$ rocking vibrations. ${ }^{30}$ In conclusion, FT-IR spectra confirmed that the reaction between the $\mathrm{OH}$ present on the silica surface and the HMDS occurred successfully, producing silica functionalized on the surface with TMS groups.

2.2. Creation of Superhydrophobic Bilayer Films by Sol-Gel Processing. The organosilica sol (named solC) used to deposit the bottom adhesive layer was prepared by an acidcatalyzed hydrolysis and condensation of methyltriethoxysilane (MTEOS): $0.753 \mathrm{~mol}(15 \mathrm{~mL})$ of MTEOS (98\%, from Fluka) was added to a stirred mixture of $0.136 \mathrm{~mol}(3 \mathrm{~mL})$ of concentrated $\mathrm{HCl}$ (from Fluka), $0.27 \mathrm{~mol}(4.86 \mathrm{~mL})$ of water, and $1.167 \mathrm{~mol}$ $(50 \mathrm{~mL})$ of ethanol $(98 \%$, from Sigma-Aldrich). This mixture was sealed and allowed to react for $90 \mathrm{~min}$ at $60^{\circ} \mathrm{C}$ and then spincoated onto the glass substrate at $3000 \mathrm{rpm}$ for $60 \mathrm{~s}$.

The acid character of solC was then neutralized by adding a certain amount of ammonium hydroxide solution $\left(28 \% \mathrm{NH}_{3}\right.$ basis, from Sigma-Aldrich) until $\mathrm{pH} 9$ was reached, and several solutions were suddenly prepared by mixing (at a volume ratio 1:1) small aliquots of the same " $\mathrm{pH} 9$ solC" to several suspensions of TMS-silica nanoparticles in MIK. The solid powder content in these suspensions was varied from 2 to $10 \%$.

All of the hydrophobic sols were stirred at $60^{\circ} \mathrm{C}$ for $24 \mathrm{~h}$ and finally spin-coated at $2200 \mathrm{rpm}$ for $60 \mathrm{~s}$ onto the previously

(28) Pozzato, A.; Dal Zilio, S.; Fois, G.; Vendramin, D.; Mistura, G.; Belotti, M.; Chen, Y.; Natali, M. Microelectron. Eng. 2006, 83, 884.

(29) Kamitsos, E. I.; Patsis, A. P.; Kordas, G. Phys. Rev. B 1993, 48, 12499.

(30) Almeida, R. M.; Pantano, G. C. J. Appl. Phvs. 1990, 68, 4225. 


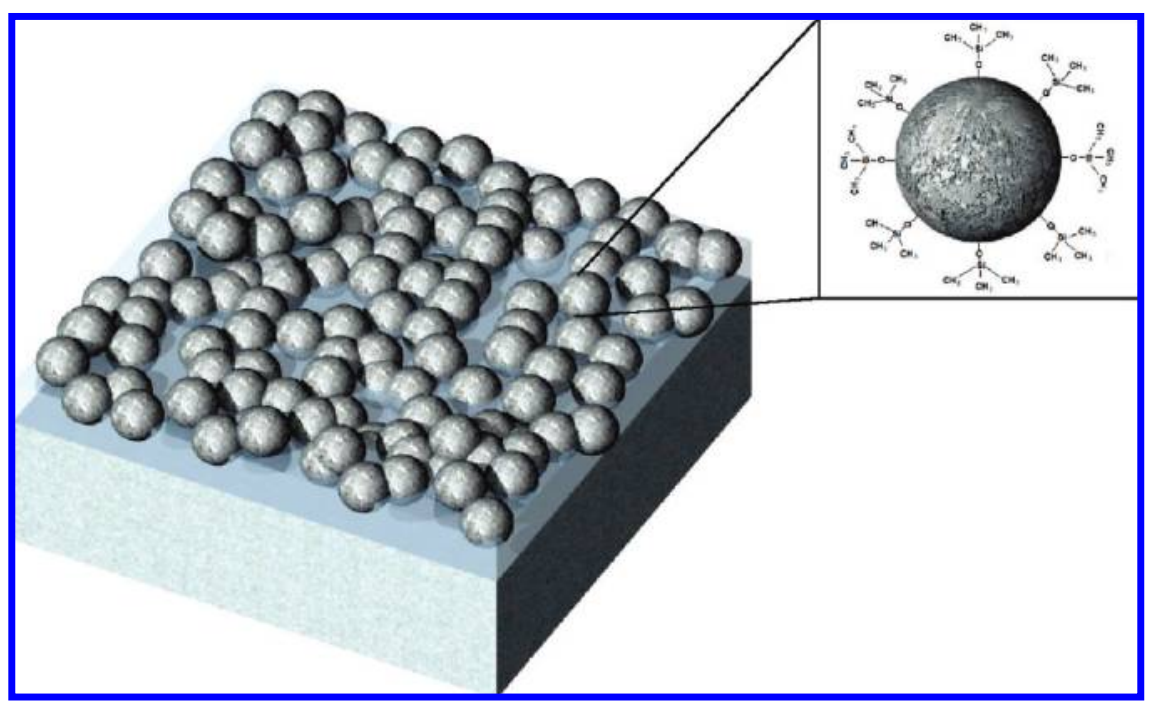

Figure 2. Preparation of a TMS-silica nanoparticles-based ultrahydrophobic coating.

deposited dense and homogeneous solC layer. Prior to treatment, all of the glass substrates were cleaned with acetone and isopropanol by sonication for $10 \mathrm{~min}$ and rinsed with deionized water. After they were coated, the samples were dried under ambient conditions for $5 \mathrm{~min}$ and then heat-treated at $350{ }^{\circ} \mathrm{C}$ in air for $30 \mathrm{~min}$.

2.3. Characterization. The surface-wetting properties were evaluated by using an OCA20 system from DataPhysics. Water CA measurements were obtained using the sessile drop method by averaging the measurements on 10 different positions of the examined surface. UV-vis spectroscopy measurements were made by a Varian Cary 5000 spectrophotometer.

The surface morphology of coated samples was characterized by both scanning electron and atomic force microscopy. In the first case, the micrographs were taken with a RAITH 150 system. An electron beam evaporator (Temescal Supersource) was used to coat a conductive layer of gold onto the silica-based films before observing the microstructure. In the second case, a SMENA MT-DTA atomic force microscope (AFM) in tapping mode was used for surface topographical characterization. All acquired AFM images were analyzed using the detection software SPIP, and several statistic parameters were used to characterize the topography. Among these, the Cassie-Baxter coefficient, denoted as the surface fraction covered by the particles aggregates, was extrapolated by performing a grain analysis with a threshold segmentation of the AFM images. In this method, the "islands" left when the image landscape is flooded to the threshold level are accounted as grains. The detection level defines the minimum $z$ value needed for a pixel to be regarded as part of a segment. To improve the detection performance, we first equalized $z$ values of our images by simply subtracting the mean value of the pixels in the local neighborhood. Then, we fixed the detection level as equal to $0.75 R_{\mathrm{y}}$. So, only pixels having a $z$ value upper this detection level were regarded, and higher hills peaks were considered. By performing this grain analysis, we were able to calculate the percentage of area covered by detected grains, that is, the surface fraction covered by the nanoparticle aggregate tops that correspond to the Cassie-Baxter coefficient.

\section{Results and Discussion}

The sol-gel process offers a convenient method to fabricate porous glass films at ambient conditions. In a typical sol-gel process, the precursor is converted into a glassy material through a series of hydrolysis and polycondensation reactions. The most important factor for the precursor solution that influences the sol-gel process is the catalyst. ${ }^{31}$ Referring to the chemistry of alkylsilanes sol-gel, it is well-known that the silica phase separation easily takes place when the $\mathrm{pH}$ value of the mixing solution is below and above the isoelectric point (IEP) of the reacting silanol. The formation of linear structures results from the acid catalyst reaction, while coagulation phenomena occur at base catalyst conditions.

Our approach utilizes an organosilica sol-gel-based doublelayer coating consisting of a first dense and homogeneous bottom layer responsible for the adhesion to the substrate and a second highly porous upper layer to which superhydrophobicity is due. A schematic description of our approach is reported in Figure 2.

First, an acid-catalyzed silica sol was prepared by using a $10 \mathrm{mM}$ ethanolic solution of MTEOS and was then depositedafter a partial gelation-by spin coating onto a precleaned glass substrate. An homogeneous and dense $180 \mathrm{~nm}$ thick film was thus obtained, which assured a strong adhesion to the glass surface. Afterward, a small amount (ranging from 2 to $10 \mathrm{wt} \%$ ) of TMS surface-functionalized silica nanoparticles (with an average diameter of 20-25 nm) was dispersed in MIK and mixed with a fixed amount of the previous prepared partially gelled acid-catalyzed silica sol.

Such a solution was spin-coated onto the first sol-gel adhesive layer, hence resulting - after the solvent evaporation-in the formation of an appropriately rough surface. The addition of such hydrophobic nanoparticles to the partially gelled silica matrix allowed the creation of a nanoporous surface with pores ranging from a few tens of nanometers up to few micrometers in diameter. By adjusting the relative content of TMS-silica nanoparticles and MTEOS-derived organosilica binder in the solution, it was possible to control the surface roughness.

Samples were then heated at $350^{\circ} \mathrm{C}$ for 30 min to allow the solgel matrix to harden. In this way, the dispersed nanoparticles were stably fixed and formed a continuous highly porous network tightly linked to the glass substrate throughout the dense highly adhesive bottom layer. On the other hand, most of the top TMS nanoparticles were only partially embedded in the underlying sol-gel film, so that they could effectively exploit their intrinsic hydrophobic behavior. The overall double-layer coating was about $400 \mathrm{~nm}$ thick.

(31) Loy, D. A.; Mather, B.; Straumanis, A. R.; Baugher, C.; Schneider, D. A.; Sanchez, A.; Shea, K. J. Chem. Mater. 2004, 16, 2041. 


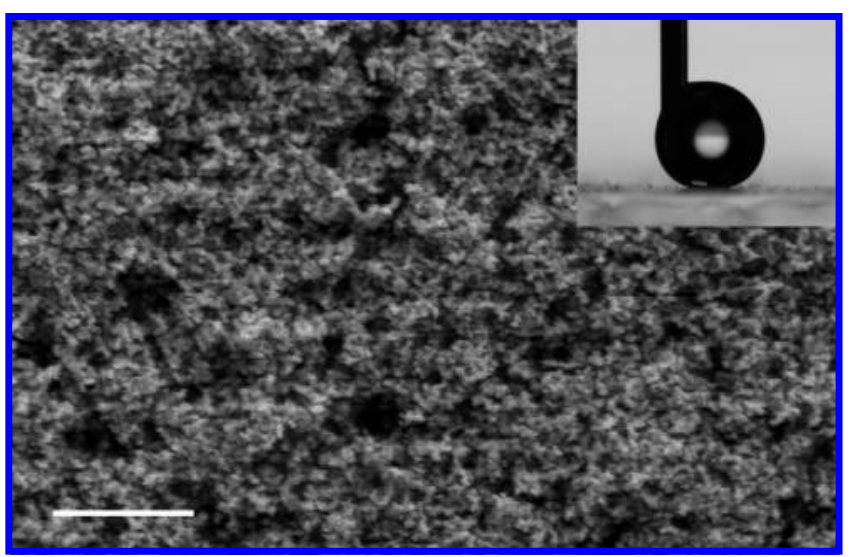

Figure 3. SEM image of a top layer obtained by spin coating of a 5 wt \% TMS-silica nanoparticles-containing solution. The inset shows a $3 \mu \mathrm{L}$ water droplet bound to the dispensing needle. Scale bar, $500 \mathrm{~nm}$.

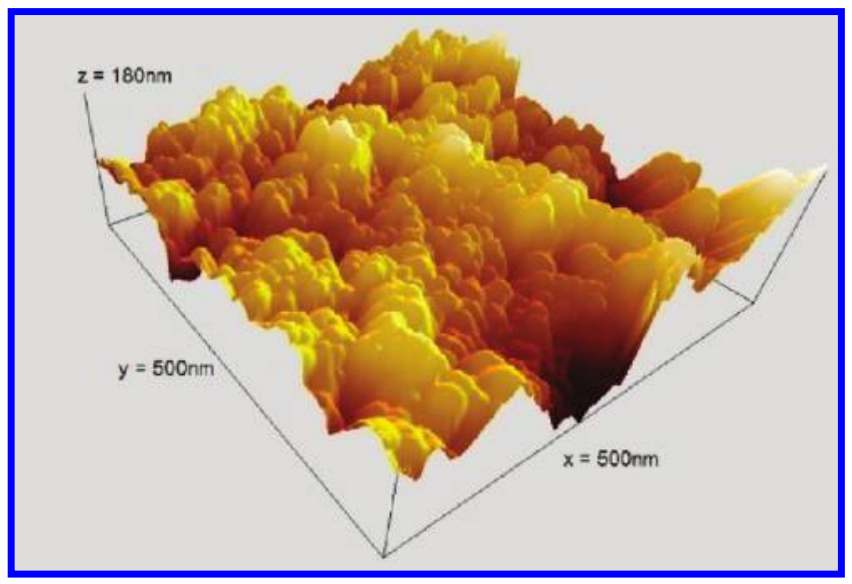

Figure 4. AFM 3D image of a top layer obtained by spin coating of a $5 \mathrm{wt} \%$ TMS-silica nanoparticles-containing solution.

A scanning electron microscopy (SEM) image of the surface topology obtained with a $5 \mathrm{wt} \%$ TMS-silica nanoparticlescontaining top layer solution is shown in Figure 3. As is clearly visible, two different levels of nanotexturing are present, being a key characteristic in the creation of superhydrophobic surfaces. The presence of protruding nubs covering the whole surface assures, in fact, that the surface contact area available to water is very low, while the hydrophobic nanoparticles prevent penetration of water into the valleys. When the coating wetability was tested by means of CA measurements, a remarkable water repellency was revealed. The water droplets rolled away immediately as they were deposited on the coated surface, even if the substrate was not appreciably tilted. This made it impossible to measure the $\mathrm{CA}$ if the droplet was not kept bound to the dispensing needle.

In the inset of Figure 3 , we reported an image of a $3 \mu \mathrm{L}$ water drop placed onto a TMS-silica nanoparticles-based bilayer coating deposited onto a glass substrate: The measured CA was found to be as high as $168^{\circ}$. As easily understood, this behavior is related both to the film morphology and to the intrinsic low surface energy of the TMS-silica particles that stem from the chemical nature of the trimethylated groups grafted onto the particles surface. These species give rise to the asymmetric molecular forces responsible for the water repulsion.

To better reveal the effect of surface texturing on ultrawater repellency, an AFM characterization (see Figure 4) has also been

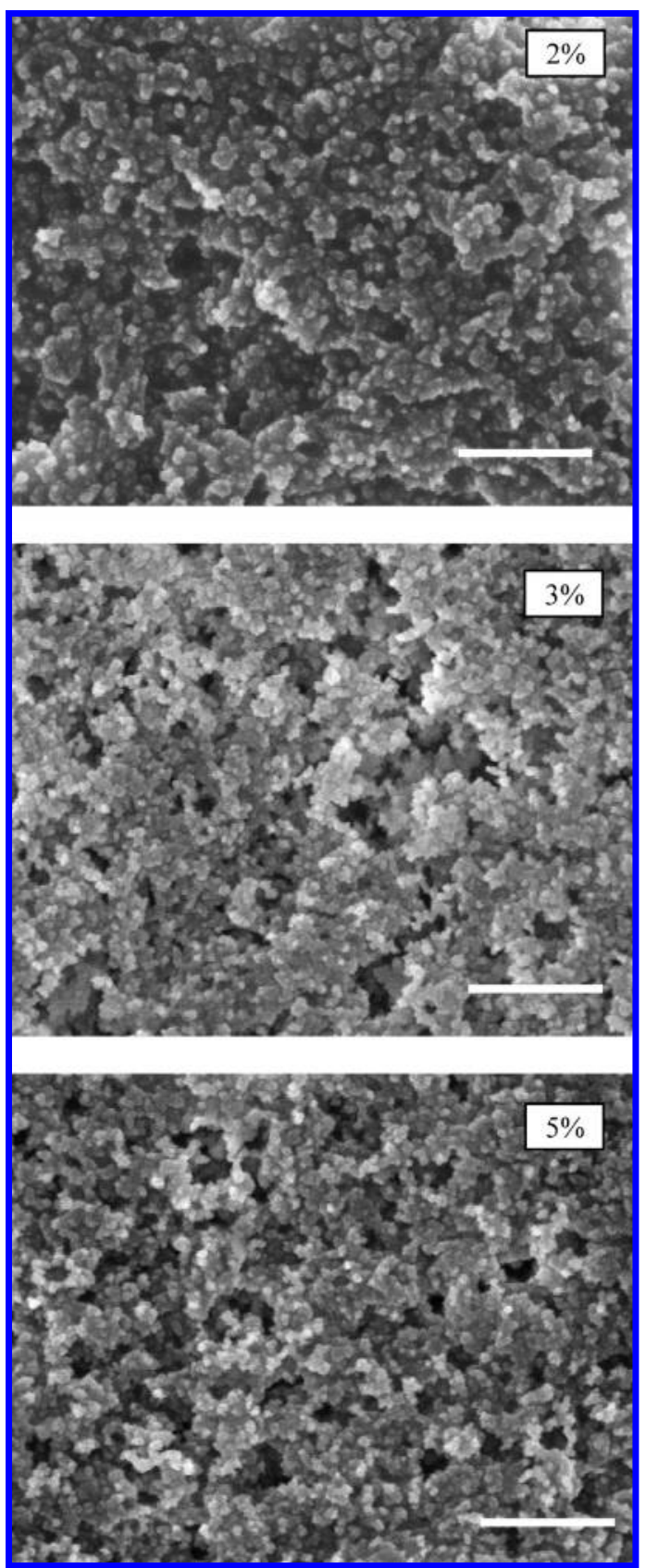

Figure 5. SEM images of top layers obtained by spin coating of 2,3 , and $5 \mathrm{wt} \%$ TMS-silica nanoparticles-containing solutions. Scale bar, $200 \mathrm{~nm}$.

done: rms roughness and the Cassie-Baxter coefficient $\varphi_{\mathrm{s}}-$ denoted as the surface fraction covered by the nanoparticles aggregates tops-were extrapolated by using the detection software SPIP. Relatively high rms values $(\sim 90-120 \mathrm{~nm})$ and intrinsically low $\varphi_{\mathrm{S}}$ values $(\sim 0.13-0.21)$ were measured, this being in perfect accordance with the nonwetting behavior of water droplets that cannot penetrate into surface cavities. This morphology results in the formation of air pockets and a composite interface under the droplet involving solid-liquid, liquid-air, and solid-air interfaces. For such a system, the theoretical CA predicted by the Cassie-Baxter model is given by: ${ }^{32}$

$$
\cos \vartheta_{\mathrm{CB}}=\varphi_{\mathrm{S}}\left(\cos \vartheta_{0}+1\right)-1
$$

(32) Cassie, A.; Baxter, S. Trans. Faradav Soc. 1944, 40, 546. 
where $\varphi_{\mathrm{S}}$ is the fraction of projected planar area of the drop in contact with the substrate and $\theta_{0}$ is the CA associated to a hypothetical flat surface having the same chemical composition of the rough one. According to eq 1 , in the limit of $\varphi_{\mathrm{S}}$ going to zero, $\theta_{\mathrm{CB}}$ approaches $180^{\circ}$.

To the aim of finding the best trade-off between superhydrophobicity and long-term durability in outdoor conditions, we realized a set of different films by tuning the relative content of

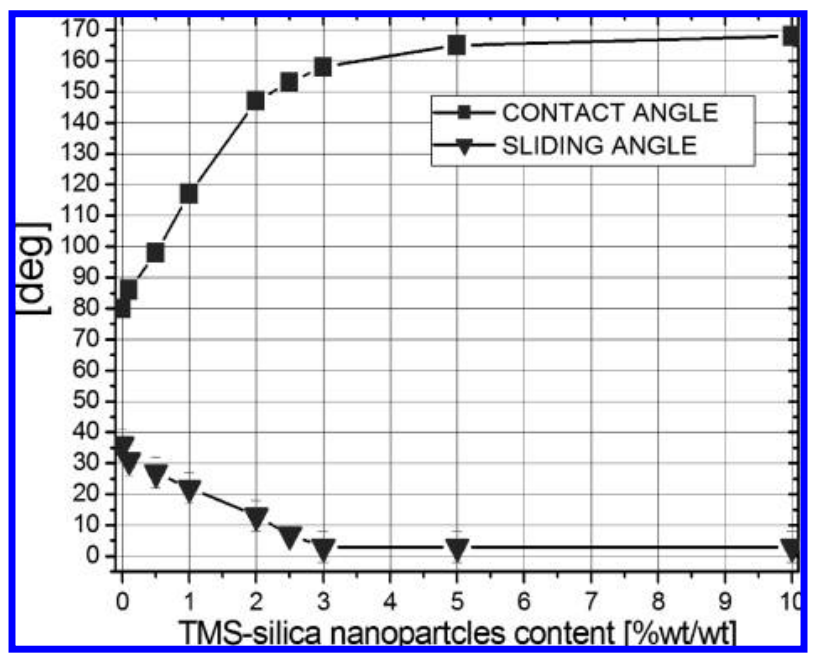

Figure 6. CAs and SAs of $3 \mu \mathrm{L}$ water droplets on surfaces coated with top layer sols having different concentrations of TMS-silica powder.

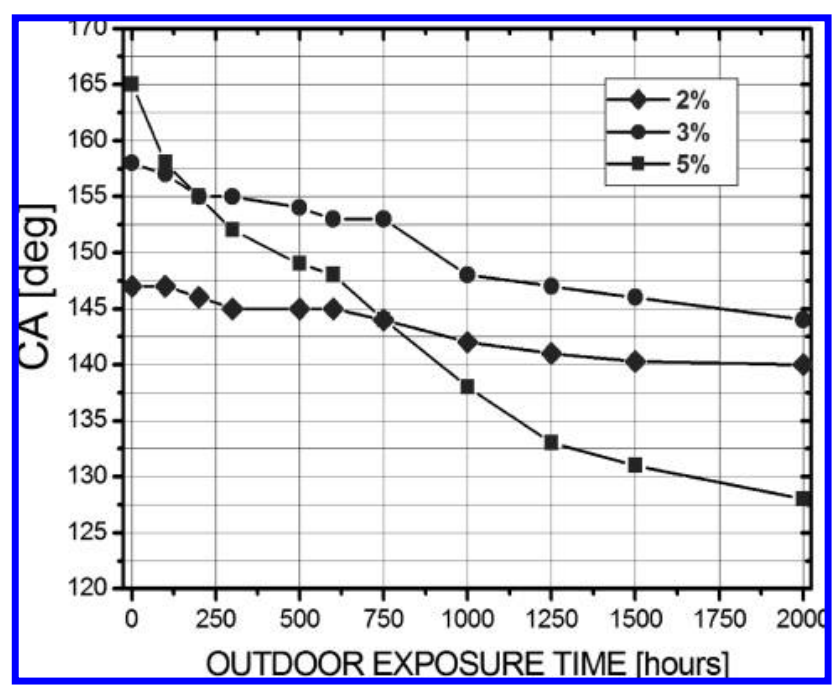

Figure 7. Changes in CA of a water droplet vs outdoor exposure time for films prepared by top layer sols having different TMSsilica powder contents.
TMS-silica nanoparticles and MTEOS-derived organosilica binder in the top-coated solution and measured their wetting properties at different time intervals during an outdoor exposure time as long as $2000 \mathrm{~h}$. Surface topographies corresponding to top layers prepared by $2 \%, 3 \%$, and $5 \%$ TMS-silica nanoparticles containing solutions are shown in Figure 5.

As reported in Figure 6, the coated surfaces began to show a superhydrophobic behavior $\left(\mathrm{CA}>150^{\circ}\right.$ and $\mathrm{CAH}<5^{\circ}$ ) when the functionalized silica powder content in the top layer sol became higher than $3 \% \mathrm{wt}$. When the amount of nanoparticles was further increased, CA approached the maximum value (of approximately $168^{\circ}$ ) allowed by the specific gravity limit of $3 \mu \mathrm{L}$ sized water droplet, and the SA became lower than $3^{\circ}$.

In Figure 7, we reported the graph of CA vs outdoor exposure time for glass samples having the same bottom layer but three different top layers (respectively obtained by solutions containing 2,3 , and $5 \mathrm{wt} \%$ of TMS-silica powder). It stands out that the durability of the self-cleaning effect was improved when the nanoparticles/binder weight ratio was decreased: In particular, a sample coated with a 3 wt \% top solution showed a CA reduction of only $12^{\circ}$ as well as a still persistent self-cleaning behavior after $2000 \mathrm{~h}$ of exposure. Rather, for higher ratios, a remarkable decrease in hydrophobicity was detected after a shorter time. This is because in the case of the low binder content in the upper layer, the on-top partially embedded TMS-silica nanoparticles could not be strongly fixed to the underlying precoated film; thus, the resulting overall superhydrophobic coating was not mechanically stable. In other terms, the higher the hydrophobic nanoparticles content in the upper layer is, the lower its strength of adhesion to the underlying silica gel base. This effect is easily explained by considering the chemical unreactivity of TMS-functionalized nanospheres that were not covalently linked to the surrounding silicate matrix but only mechanically embedded within it. As a consequence, if the number of particles exceeds the maximum amount of binder necessary to effectively embed them, the coating's durability results are compromised. In Figure 8, a surface coated with a 5 wt \% TMS-silica nanoparticles containing solution is reported after $2000 \mathrm{~h}$ exposed at environmental conditions. As clearly visible, it resulted in wide damage and revealed the presence of extended zones in which nanoparticles were totally missing.

A fine trade-off between superhydropohobicity and coating long-term durability was found when the nanoparticles content in the sol was kept in the range between 2 and 3\% wt. In addition, it has to be underlined that if any additional fluorinated capping layer was used after the annealing treatment at $350{ }^{\circ} \mathrm{C}$, this lead to huge beneficial effects in terms of thermal stability. The intrinsic surface hydrophobicity is in fact due to the presence of a hydrocarbon side group $\left[-\mathrm{Si}\left(\mathrm{CH}_{3}\right)_{3}\right.$ onto the silica nanoparticles and $\equiv \mathrm{Si}-\mathrm{CH}_{3}$ groups within the organosilica matrix] that start to decompose at elevated temperatures (just below $400{ }^{\circ} \mathrm{C}$ ).

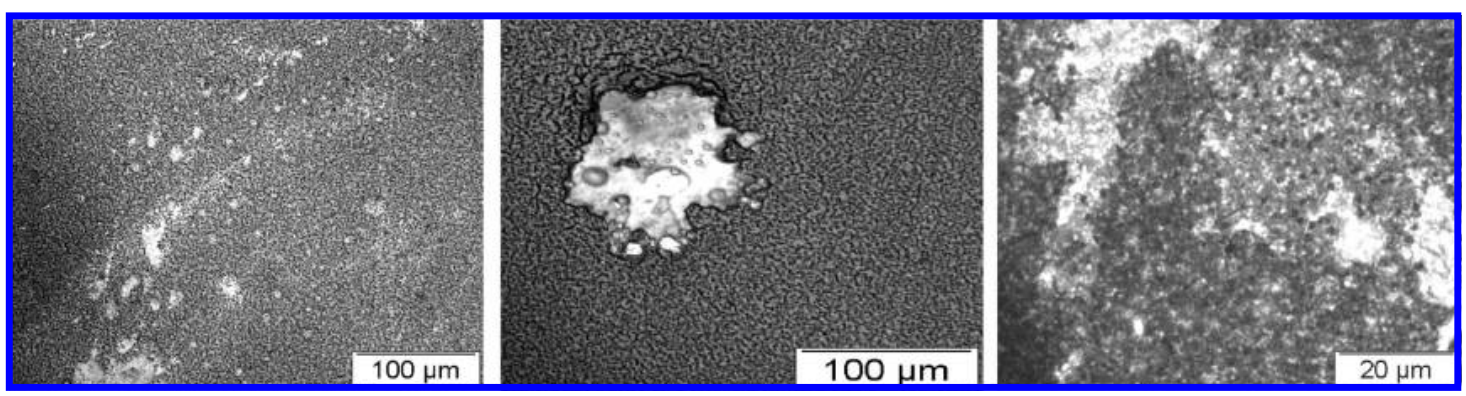

Figure 8. Optical images of a glass surface coated with a $5 \mathrm{wt} \%$ TMS-silica nanoparticles top layer after $2000 \mathrm{~h}$ of outdoor exposure. 


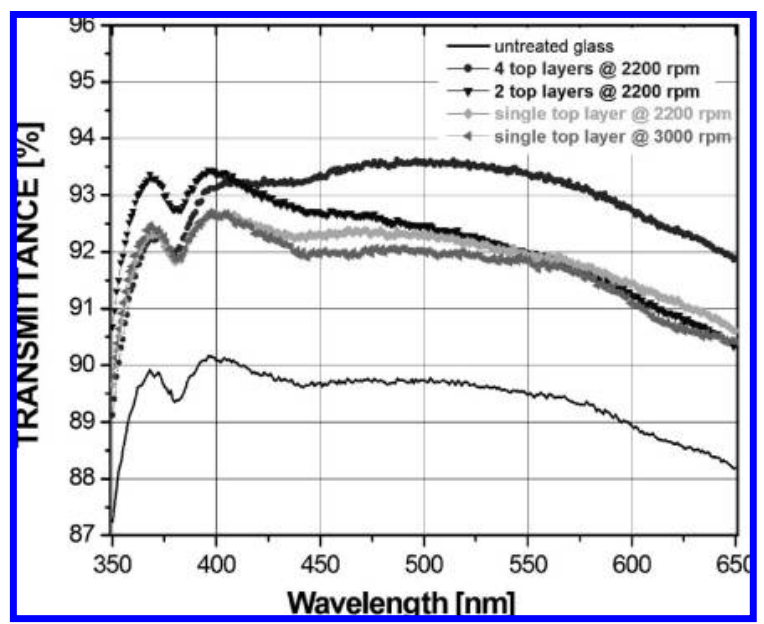

Figure 9. Transmission spectra of glass plates coated with $3 \mathrm{wt} \%$ TMS-silica nanoparticles-containing top layer solutions deposited at different spin-coating rates.

Finally, optical characteristics of such coated superhydrophobic surfaces have also been tested by means of transmittance measurements. In Figure 9, the transmission spectra of several coated samples are reported and compared to the spectrum of an uncoated sample. As well-known, a nanoporous film acts as an homogeneous film having an effective refractive index lower than its corresponding bulk material ${ }^{33}$ so as to produce a consistent antireflection effect. As compared to an uncoated glass plate, when coated with a $3 \mathrm{wt} \%$ TMS-silica nanoparticles-containing top layer solution, surfaces exhibited, in fact, fairly reduced reflective losses throughout all of the visible region. To minimize such losses, we tuned the thickness of such nanoporous top layer by regulating the spin-coating speed or by depositing more than one top layer. The best performances were obtained when four nanoporous top layers were spin-coated (onto the precoated homogeneous sol-gel layer) at $2200 \mathrm{rpm}$ : An increase of more than $3 \%$ in transmission within all of the visible range and a

(33) Yoldas, B. E.; O’Keeffe, T. W. Appl. Opt. 1979, 18, 313.

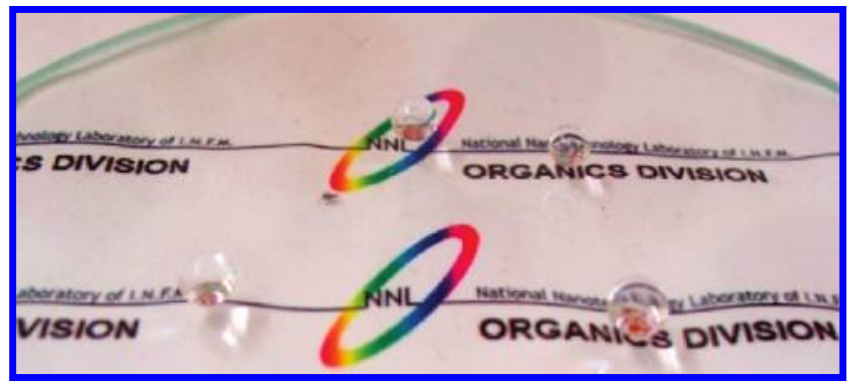

Figure 10. Image of a glass plate coated with a superhydrophobic antireflective bilayer coating.

highest absolute transmittance of $93.6 \%$ at $500 \mathrm{~nm}$ were observed. Figure 10 shows the picture of a glass plate coated with an optimized superhydrophobic highly transparent coating obtained by the presently proposed method.

\section{Conclusions}

In summary, we successfully developed a sol-gel-based method to generate robust superydrophobic and-simultaneouslyantireflective surfaces. By employing TMS surface-functionalized silica nanoparticles partially embedded into an organosilica gel matrix, we were able to construct solid surfaces having both appropriate roughness and low surface energy without any additional hydrophobic capping layer. We demonstrated that, by tuning the relative content of hydrophobic nanoparticles and silica gel binder, it is possible to optimize both the water repellence and the thermomechanical stability of the coated surfaces. The nanoporous nature of the upper layer also gave rise to a consistent antireflection effect. It is envisaged that, because of the simplicity and effectiveness of this procedure, it can be employed in a wide variety of large-scale both industrial and scientific applications.

Acknowledgment. This work has been partially supported by iGUZZINI ILLUMINAZIONE SpA [Recanati (MC), Italy] as an industrial partner. We thank Francesco Quercetti and Massimo Gattari for beneficial discussions. 